\title{
SMR
}

\section{Management of clinically negative nodes (NO) in supraglottic laryngeal carcinoma: A systematic review}

\author{
Y.H. Liu and Z.W. Du \\ Department of Otorhinolaryngology Head and Neck Surgery, \\ Second Affiliated Hospital of Nanchang University, Nanchang, China \\ Corresponding author: Y.H. Liu \\ E-mail: yuehuiliudoc@163.com
}

Genet. Mol. Res. 15 (4): gmr15048179

Received December 17, 2015

Accepted January 15, 2016

Published October 17, 2016

DOI http://dx.doi.org/10.4238/gmr15048179

Copyright (C) 2016 The Authors. This is an open-access article distributed under the terms of the Creative Commons Attribution ShareAlike (CC BY-SA) 4.0 License.

ABSTRACT. The purpose of this study was to evaluate the treatment
of clinically negative cervical lymph nodes in supraglottic carcinoma
by a meta-analysis. The search words were "supraglottic carcinoma",
"cervical lymph nodes negative/cN0", "radical neck dissection",
and "radiotherapy". The databases included the Chinese biomedical
literature database, Medline, Cochrane library, EMBASE database,
journals, and theses, etc. from 1989 onwards. Using the 5-year overall
survival, disease-free survival, and disease-specific survival rates, and
the recurrence and distant metastasis rates as observation indexes, the
proper model and method were selected after a heterogeneity test to
allow combined statistic tests, sensitivity analysis, and publication
bias analysis to be conducted. Four studies ( 807 cases) were included
in the analysis. Comparisons of the 5-year overall survival, disease-
free survival, and disease-specific survival rates as well as lymph
node metastasis and the recurrence rate for radical neck dissection

Genetics and Molecular Research 15 (4): gmr15048179 
and radiotherapy showed no significant differences. There was no advantage of radical neck dissection in supraglottic carcinoma with clinically negative cervical lymph nodes compared to radiotherapy. However, owing to the lack of a prospective study and large number of cases, selection bias and measurement bias may still exist.

Key words: Supraglottic carcinoma; Clinically negative node; Meta-analysis; Radical neck dissection; Radiotherapy

\section{INTRODUCTION}

Head and neck cancers are the sixth most common cancer worldwide. It has been estimated that a total of 53,640 new head and neck (oral cavity, pharynx, and larynx) cancer cases including 11,520 deaths were observed in the United States in 2013 (Siegel et al., 2013), In Australia, 584 cases of laryngeal cancer were reported in 2001, and it accounted for 247 deaths in the same year (Australian Institute of Health and Welfare website: http://www.aihw. gov.au/publications/can/ca01/ca01.pdf). Since laryngeal cancers account for about one-quarter of all head and neck cancers, and overall survival (OS), disease-free survival (DFS), and larynx reservation are largely determined by the initial tumor stage at the time of diagnosis (American Cancer Society, 2013), while the rate of occult metastasis of supraglottic carcinoma lymph nodes was 30\% (Redaelli de Zinis et al., 1994). The supraglottic area is rich in lymphoid tissue. In addition, the laryngeal ventricle region is a transitional area of quadrangular membrane and the conus elasticus, and lacks the protection of elastic fibers, which causes invasion of the paraglottic space extremely easy. In addition, the clinical experience of neck palpation and clinical examination is limited, and auxiliary image examination is often unable to detect small lesions.

In "conventional" open transcervical surgical resection of lesions or tumors of the upper aerodigestive tract, visible mutilation often occurs with unfavorable aesthetic and functional results, in particular, failures to swallowing, breathing, and voice disorders (Arens, 2012). The choice of treatment depends on staging and histology (Tantiwongkosi et al., 2014). It has been shown that supraglottic laryngectomy is an effective approach for supraglottic carcinoma in the early stages, while radiotherapy is an accepted alternative to surgery in early stage disease, especially in elderly and multimorbid patients with pulmonary dysfunction, because of significant morbidity and postoperative functional problems after classic partial supraglottic laryngectomy (Canis et al., 2013). In N0 patients with laryngeal cancer, unilateral or bilateral elective neck dissection should be performed according to the anatomical location and histopathological specimens should be taken in the event of positive detection of metastases concluding that postoperative radiotherapy should be performed (Mutlu et al., 2014).

Traditionally, the treatment for laryngeal cancer has included total laryngectomy and radiotherapy, used either alone or in combination. There have been significant technological advances in voice rehabilitation, delivery of radiation as well as development and popularization of conservative surgical techniques (Chawla and Carney, 2009). At present, the treatment methods for clinically negative nodes (cN0) supraglottic carcinoma remain controversial. Some perform positive cervical lymphadenectomy, and some do conservative radiotherapy (Pillsbury and Clark, 1997). However, studies of effectiveness lack a large number of cases, a prospective study, and a control study. Therefore, it is necessary to perform a comprehensive analysis of the existing clinical trials to provide evidence for clinical treatment.

Genetics and Molecular Research 15 (4): gmr15048179 


\section{MATERIAL AND METHODS}

\section{Study types}

The trials were randomized controlled trials or quasi-randomized controlled trials, which have been published. In the literature, there were clear censored follow-up data or survival curves.

\section{Observation objects}

All cases were confirmed by pathology or cytology for supraglottic carcinoma, and lymph node cervical metastasis was not detected on clinical examination (including clinical palpation, B ultrasound, computerized tomography, etc.) indicating $\mathrm{cN} 0$. The age, gender, region, and race of patients were not limited.

\section{Intervention measures}

For cN0 cervical lymph nodes in supraglottic laryngeal carcinoma, the test group underwent radical neck dissection and the control group underwent radiotherapy.

\section{Curative effect indexes}

The curative indexes were 5-year OS, 5-year disease-specific survival rate (DSS), 5-year DFS, recurrence rate, and distant metastasis rate.

\section{Retrieval strategies}

Online retrieval scope: MEDLINE, OVID, EMBASE, Cochrane laboratory, Chinese Biomedical Literature Database, etc. There was no language limitation. The retrieval time ranged from database establishment to September 2012. The language was limited to Chinese and English.

\section{Screening and quality evaluation of literature}

On reading the document title and abstract, tests that obviously did not meet the inclusion criteria were discarded. Then, the full text of the tests that might meet the inclusion criteria were read to determine whether the tests were really in accordance with the inclusion criteria. In the absence of a quality gold standard, the evaluation of non-randomized controlled trials used "non-randomized clinical trial quality evaluation criteria and the evaluation score table" of the Chinese Evidence-Based Medicine Center. Evaluations were carried out from 6 aspects that included grouping method, blind method, inclusive analysis of the studied cases, research baseline, diagnostic criteria, and control for confounding factors (Liu, 2001).

\section{Statistical analysis}

Data processing and analysis was performed using the Revman4.3 software provided by Cochrane collaboration net. Extraction of all eligible data in the literature was on the basis 
of the results indexes. The Revman 4.3 software can be used to judge the heterogeneity of the included studies using the $\mathrm{P}$ value of the heterogeneity test result shown on a Forest graph. If $\alpha$ $=0.05$ and $\mathrm{P}>0.05$, more studies were concluded to be homogeneous. The Peto method was used to calculate the combined statistics by the fixed effects model. If $\mathrm{P} \leq 0.05$, we concluded that more similar studies were heterogeneous. We then used the Dersimonian-Laird method to calculate merged statistics using the random effect model after correction. The results indexes in this study were two categorical variables, and the odds ratio (OR) was used as the combined statistics. The Revman 4.3 software could calculate the $95 \%$ confidence interval $(95 \% \mathrm{CI})$ of the combined statistics shown in the Forest graph; when $\mathrm{OR}=1$, the test effect was invalid, and if the $95 \% \mathrm{CI}$ contained 1, which was equivalent to $\mathrm{P}>0.05$, there was no statistical significance; if the upper and lower limit did not contain 1 (greater than 1 or less than 1), which was equivalent to $\mathrm{P}<0.05$, there was statistical significance.

\section{RESULTS}

\section{Retrieval of results}

Literature was retrieved online according to the retrieval strategy, the document title and abstract were screened in strict accordance with the inclusion and exclusion criteria, the document title and abstract were read, and the tests that obviously did not meet the inclusion criteria were excluded. Then, the full text of the tests that might meet the inclusion criteria were read to determine whether the tests were really in accordance with the inclusion criteria. A total of 4 studies were selected, in which 1 involved domestic research (Zhang, 2009) and 3 foreign research (Spriano et al., 1997; Orús et al., 2000; Sessions et al., 2005), including 807 patients. Basic conditions of each included clinic study are shown in Figure 1.

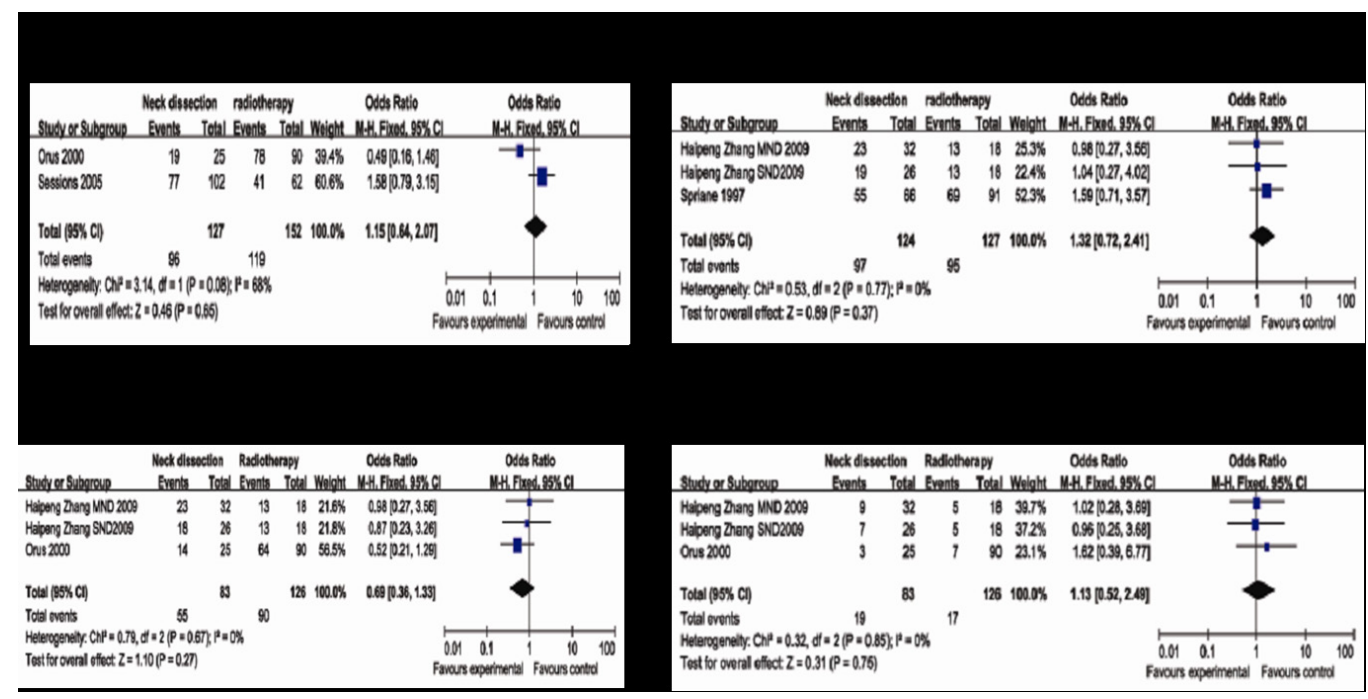

Figure 1. Forest plot of comparison of neck dissection $v s$ radiotherapy. A. Disease-specific survival rate at 5 years; B. Disease-free survival rate at 5 years; C. Overall survival rate; D. Recurrence rate. 


\section{Quality evaluation of included literature}

The 4 articles were all retrospective case-control studies. Therefore, we evaluated the included literatures considering the grouping method, blind method, whether all of the studied cases were included in the analysis, research baseline, diagnostic criteria, and control of confounding factors. The quality scores of each of the included studies are shown in Figure 1.

\section{Five-year DSS rate}

There were two reports about the 5-year DSS rate. In the Session's report (Sessions et al., 2005), the DSS rate for the radical neck dissection group was $75.5 \%(95 \% \mathrm{CI} 66.3-83.8)$ and the radiotherapy group was $66.1 \%(95 \% \mathrm{CI} 53.7-76.7)$. The rate difference was $+9.4 \%(95 \% \mathrm{CI}$ $=-4.6 \%$ to +23.8$)$, while in reports by Orús et al. (2000) the DSS rates for the two groups were $78 \%(95 \% \mathrm{CI}=60-96)$ and $87 \%(95 \% \mathrm{CI}=79-95)$ respectively. The rate difference was $-10.8 \%$ $(95 \% \mathrm{CI}=-31.0$ to +4.3$)$. The $\mathrm{OR}$ values and $95 \% \mathrm{CI}$ exhibited $\mathrm{P}>0.05$, being then combined with fixed-effect model. After the combination, $\mathrm{OR}=1.15(95 \% \mathrm{CI}=0.64-2.07, \mathrm{P}=0.65)$ showed no significant difference. Radical neck dissection and radiotherapy showed no significant difference in the 5-year DSS rates among cN0 patients with supraglottic carcinoma (Figure 1A).

\section{Five-year DFS rate}

There were reports on the 5-year DFS rate. In the report by Spriano et al. (1997), DFS of the selective neck dissection group was $75.5 \%(95 \% \mathrm{CI}=66.3-83.8)$, and that of the radiotherapy group was $66.1 \%(95 \% \mathrm{CI}=53.7-76.7)$, while in reports by Zhang (2009), DFS of the selective neck dissection group, modified radical neck dissection group, and radiotherapy group was $73.1,71.9,72.2 \%$, respectively. The two groups' OR and $95 \%$ CI were calculated. The heterogeneity test showed $\mathrm{P}>0.05$ and combined using a fixed-effect model. After combining, $\mathrm{OR}=1.32(95 \% \mathrm{CI} 0.72-2.41), \mathrm{P}=0.37$, which showed that there were no significant difference. Neck dissection groups and the radiotherapy group had no significant difference in 5-year DFS rates of cN0 patients with supraglottic carcinoma (Figure1B).

\section{Overall survival rate}

There were two reports on the OS rate. In the reports by Orús et al. (2000) and Spriano et al. (1997), OS in the selective neck dissection group was 78\% $(95 \% \mathrm{CI}=60-96 \%)$, and that in the radiotherapy group was $87 \%(95 \% \mathrm{CI}=79-95 \%)$, while in Zhang (2009) reports, the OS rates of the selective neck dissection, modified radical neck dissection, and radiotherapy groups were $70.3,72.5$, and $72.6 \%$. The two groups' OR and $95 \% \mathrm{CI}$, heterogeneity test showed $\mathrm{P}>0.05$ and combined using a fixed-effect model, after combining, $\mathrm{OR}=0.69,95 \% \mathrm{CI}=0.36$ 1.33 , and $\mathrm{P}=0.27$, which showed that there was no significance difference. Neck dissection (modified and selective) and radiotherapy showed no significant difference in 5-year DSS rate of cN0 patients with supraglottic carcinoma (Figure 1C).

\section{Distant metastasis and recurrence}

There were two reports on distant metastasis and recurrence. In the reports by Orús et

Genetics and Molecular Research 15 (4): gmr15048179 
al. (2000) and Spriano et al. (1997), distant metastasis and recurrence rate in the selective neck dissection group was $12 \%$, and that in the radiotherapy group was $7.8 \%$, while in reports by Zhang (2009), distant metastasis and recurrence rates of the selective neck dissection group, modified radical neck dissection group, and radiotherapy groups were 26.9, 28, and 27.7\%, respectively. The two groups' OR and 95\%CI were calculated. The heterogeneity test showed $\mathrm{P}>0.05$ and combined using the fixed-effect model. After combining, the $\mathrm{OR}=1.13,95 \% \mathrm{CI}$ $=0.52-2.49, \mathrm{P}=0.75$, which showed that there were no significant differences. The neck dissection (modified and selective) and radiotherapy groups showed no significant difference in 5-year DSS rates of $\mathrm{cN} 0$ patients with supraglottic carcinoma (Figure 1D).

\section{DISCUSSION}

A randomized controlled trial is the best method to evaluate the intervention treatment effect and to keep the study results free from bias. However, since the surgical operation characteristic itself including many human factors and high operability, peri-operation period, and postoperative treatment might also affect the outcome of surgery, randomized controlled trials of surgeons are difficult to carry out (McLeod, 1999).

A total of 4 studies were selected in this study, wherein 1 involved domestic research and 3 involved foreign researchers and included 807 patients. The studies were uneven in quality, which was not suitable for the Jadad standard. The argument intensity was not high because the non-randomized controlled trials did not adopt the blind method and the grouping method was mechanical. In addition, because of non-random grouping, bias between the groups was probable. The NRCT (non-randomized controlled trials) score standard was not unified, the evaluation of NRCT used the "NRCT quality evaluation criteria and evaluation score table" of the Chinese Evidence-Based Medicine Center. The scores of the 4 articles were not high, but the heterogeneity test showed that homogeneity between groups was better, and most of the meta-analyses used the fixed-effect model, then combined them (the value from the fixed-effect model) to calculate the OR and P values, and 95\%CI.

There is still controversy about the choice of treatment methods for $\mathrm{cN} 0$ supraglottic laryngeal carcinoma. The treatment of cervical lymph nodes has been a particular focus of the debate. This is determined by its anatomical structure. The supraglottic tissues have a rich lymphatic network and easily cause occult metastasis. Besides, the current clinical examination method has limitations, which are the main reasons of treatment failure (Parmar et al., 1998). Ji et al. (2001) and Li et al. (2013) found that cN0 cervical lymph nodes in supraglottic carcinoma metastasis were mainly located in areas II, III, IV, which accounted for $96 \%$ of cases, and areas I and IV were rarely involved. In 1999, the Brazilian Head and Neck Cancer Group published the results of a randomized study of 132 cases of selective neck dissection $v s$ modified radical neck dissection in the management of $\mathrm{cN} 0$ supraglottic and transglottic carcinomas, which showed that the two groups' cervical lymph node metastasis rates were 4.8 and $2.5 \%$, respectively. The statistical analysis showed no significant difference between the two groups (Brazilian Head and Neck Cancer Study Group, 1999). Selective neck dissection has significantly improved the quality of life in patients. However, in a clinical experiment comparing the treatments of N0 supraglottic carcinoma lymph nodes in 807 patients, the differences between radical neck dissection and radiotherapy were still inconclusive. This study expected to identify the best treatment method to guide clinical practice through analyzing four clinical treatment methods. The surgery outcome was always related to the

Genetics and Molecular Research 15 (4): gmr15048179 
degree of neurological deficit, while the determinants of the prognosis of radiotherapy were residual tumor and tumor metastasis. Hypoxia is a common feature of tumors, and contributes to the development of tumorous aggression and metastasis, and reportedly plays a key role in radioresistance, chemoresistance, and poor prognosis (Li et al., 2012).

The limitations of this study are the poor quality of the included literature and small sample size for which the publication bias cannot be evaluated. In addition, each document measurement index was different, leading to difficulty in combined data analysis. Moreover, some articles could not be included, leading to selection bias. Because of all the different characteristics of the included literature, this article could not do a sensitivity test using stratified analysis, which might have led to a results bias.

The meta-analysis results showed that among the treatment methods for cervical lymph nodes in cN0 supraglottic laryngeal carcinoma, radical neck dissection, radiotherapy, comprehensive treatments, and clinical observation showed no significant difference in DFS, OS, lymph node metastasis, and recurrence of DSS. However, because of the small sample size and inclusion of non-randomized controlled trials, bias still existed. Furthermore, because evidence-based surgery itself has limitations, there is inadequate evidence to identify the pros and cons of various methods, and many unsolved problems still exist. Therefore, at this stage, there is an urgent need for large sample, multi-center, prospective, randomized, and controlled research.

\section{Conflicts of interest}

The authors declare no conflict of interest.

\section{REFERENCES}

American Cancer Society (2013). Cancer facts \& figures: 2013. American Cancer Society, Atlanta.

Arens C (2012). Transoral treatment strategies for head and neck tumors. GMS Curr. Top. Otorhinolaryngol. Head Neck Surg. 11: Doc05.

Brazilian Head and Neck Cancer Study Group (1999). End results of a prospective trial on elective lateral neck dissection $v s$ type III modified radical neck dissection in the management of supraglottic and transglottic carcinomas. Head Neck 21: 694-702. http://dx.doi.org/10.1002/(SICI)1097-0347(199912)21:8<694::AID-HED3>3.0.CO;2-B

Canis M, Martin A, Ihler F, Wolff HA, et al. (2013). Results of transoral laser microsurgery for supraglottic carcinoma in 277 patients. Eur. Arch. Otorhinolaryngol. 270: 2315-2326. http://dx.doi.org/10.1007/s00405-012-2327-6

Chawla S and Carney AS (2009). Organ preservation surgery for laryngeal cancer. Head Neck Oncol. 1: 12-15. http:// dx.doi.org/10.1186/1758-3284-1-12

Li A, Liang H, Li W, Wang Z, et al. (2013). Spectral CT imaging of laryngeal and hypopharyngeal squamous cell carcinoma: evaluation of image quality and status of lymph nodes. PLoS One 8: e83492. http://dx.doi.org/10.1371/ journal.pone.0083492

Li JZ, Gao W, Chan JY, Ho WK, et al. (2012). Hypoxia in head and neck squamous cell carcinoma. ISRN Otolaryngol. 2012: 708974. http://dx.doi.org/10.5402/2012/708974

Liu J (2001). Evaluation of the system of non-randomized studies. Chinese Journal of Evidence-Based Medicine 1: 239243.

McLeod RS (1999). Issues in surgical randomized controlled trials. World J. Surg. 23: 1210-1214. http://dx.doi. org/10.1007/s002689900649

Mutlu V, Ucuncu H, Altas E and Aktan B (2014). The relationship between the localization, size, stage and histopathology of the primary laryngeal tumor with neck metastasis. Eurasian JMed 46: 1-7. http://dx.doi.org/10.5152/eajm.2014.01

Orús C, León X, Vega M and Quer M (2000). Initial treatment of the early stages (I, II) of supraglottic squamous cell carcinoma: partial laryngectomy versus radiotherapy. Eur. Arch. Otorhinolaryngol. 257: 512-516. http://dx.doi. org/10.1007/s004050000276

Genetics and Molecular Research 15 (4): gmr15048179 
Parmar MKB, Torri V and Stewart L (1998). Extracting summary statistics to perform meta-analyses of the published literature for survival endpoints. Stat. Med. 17: 2815-2834. http://dx.doi.org/10.1002/(SICI)10970258(19981230)17:24<2815::AID-SIM110>3.0.CO;2-8

Pillsbury HC, 3rd and Clark M (1997). A rationale for therapy of the N0 neck. Laryngoscope 107: 1294-1315. http:// dx.doi.org/10.1097/00005537-199710000-00004

Redaelli de Zinis LO, Nicolai P, Barezzani MG, Tomenzoli D, et al. (1994). Incidence and distribution of lymph node metastases in supraglottic squamous cell carcinoma: therapeutic implications. Acta. Otorhinolaryngol. Ital. 14: 19-27.Sessions DG, Lenox J and Spector GJ (2005). Supraglottic laryngeal cancer: analysis of treatment results. Laryngoscope 115: 1402-1410.

Ji W, Yu J and Guan C (2001). Pathologic features of occult lymphatic metastasis in supraglottic carcinoma. Chin. Med. J. (Engl.) 114: 88-89.

Sessions DG, Lenox J and Spector GJ (2005). Supraglottic laryngeal cancer: analysis of treatment results. Laryngoscope 115: $1402-1410$

Siegel R, Naishadham D and Jemal A (2013). Cancer statistics, 2013. CA Cancer J. Clin. 63: 11-30. http://dx.doi. org/10.3322/caac. 21166

Spriano G, Antognoni P, Piantanida R, Varinelli D, et al. (1997). Conservative management of T1-T2N0 supraglottic cancer: a retrospective study. Am. J. Otolaryngol. 18: 299-305. http://dx.doi.org/10.1016/S0196-0709(97)90023-5

Tantiwongkosi B, Yu F, Kanard A and Miller FR (2014). Role of (18)F-FDG PET/CT in pre and post treatment evaluation in head and neck carcinoma. World J. Radiol. 6: 177-191. http://dx.doi.org/10.4329/wjr.v6.i5.177

Zhang H (2009). Dissection in the management of the clinically negative neck (N_0) supraglottic laryngeal squamous cell carcinoma. Available at [http://cdmd.cnki. com.cn/Article/CDMD-10114-2009123560.htm].

Genetics and Molecular Research 15 (4): gmr15048179 